\title{
ECT: Examining a controversial therapy in the armamentarium of psychiatry
}

\author{
Nirushan Puvanenthirarajah, Asma Amir Ali \\ Faculty Reviewer: Shelley McKellar, PhD (Department of History)
}

\begin{abstract}
Historically, treatments for severe psychoses and affective disorders were nonexistent, and patients with mental disorders were transferred to asylums for public safety. This deficiency in treatment inspired the inception of somatic therapies, of which electroconvulsive therapy (ECT) was the most efficacious. This paper will outline the birth of ECT, the controversies leading to its decline, and the subsequent resurgence back into practice.
\end{abstract}

\section{INTRODUCTION}

Before the 20th century, psychiatry as a field of medicine offered little to individuals suffering from severe psychoses or affective disorders. Treatments for mental illness were scarce, the public perceived the mentally ill as dangerous, and individuals with mental disorders were involuntarily transferred from their homes into dirty, overcrowded public institutions, supposedly for treatment and public safety. ${ }^{1,2}$ Individuals who experienced mild psychotic episodes were typically locked into isolation and, if the episodes became too severe, they received sedation to control their behaviour. ${ }^{3}$ Since few individuals could afford the handful of private asylums that existed, most patients were admitted to public institutions, referred to as insane or lunatic asylums. These were harsh, ill-equipped facilities with underpaid staff who often treated patients cruelly. ${ }^{2}$

The first feasible breakthrough in treating psychiatric illnesses arose from advancements in psychodynamic therapies, notably the work of Sigmund Freud. Although this approach proved fruitful for neuroses and milder mental disturbances, treatment development for individuals suffering more severe psychoses remained stagnant. ${ }^{1,4}$ This impasse in the treatment of mental illness sparked the inception of somatotherapies - treatments by chemical or physical means. ${ }^{5}$ During the early decades of the 20th century, 3 unsubstantiated procedures were proposed as methods to shock the patient out of illness: insulin shock therapy, chemical convulsion shock therapy, and electroconvulsive shock therapy. ${ }^{5}$ Today, only electroconvulsive therapy remains in the armamentarium of psychiatrists, but not without its share of controversy.

\section{DISCOVERY OF ECT AND EARLY APPLICATION}

Lacking modalities for treating severe psychoses, Hungarian physician Ladislaus von Meduna turned to the medical histories and autopsies of mentally ill patients in search of a solution. ${ }^{5}$ What came of this search was the discovery that schizophrenia and epi- lepsy seldom occurred together, a phenomenon he coined biological antagonism. ${ }^{5}$ This phenomenon set the foundation for a belief that inducing convulsions could be used as a therapy for psychotic disorders. Stimulating convulsions through metrazol, a pharmacologic agent, Meduna showed the potential of convulsive shock in reducing psychosis symptoms. ${ }^{5}$ But there was a disturbing response in witnessing conscious patients endure painful convulsions that reduced the appeal of this particular treatment. ${ }^{3}$

On the heels of Meduna's discovery, neurologist Ugo Cerletti extended the induction of convulsion to electric shocks. Having specialized in epilepsy, Cerletti understood that electric shocks applied to the cranium of pigs would create a transient epilepsy-like convulsion. ${ }^{5}$ In contrast to pharmacological induction of convulsions, electrical induction had the added benefit of retrograde amnesia, which increased its desirability as a treatment since patients would not remember the pain associated with convulsion. ${ }^{4}$ Similar to pharmacologic convulsion therapy, ECT reduced symptoms in patients suffering from depression compared to other treatments. ${ }^{4}$

The transition of electroshock therapy from animal models to human subjects raised serious ethical problems. The first test on a human patient occurred in April of 1938 when patient S.E., who had been diagnosed as paranoid schizophrenic, was institutionalized against his will and, as doctors later recounted, received treatment without understanding what was about to happen. ${ }^{5}$ S.E. later published a letter retroactively praising the doctors for curing him of his illness, which instilled the belief that convulsive shock could be used to treat mental illness in humans. ${ }^{5}$ The immediate improvement and short-term convalescence observed in this patient and a handful of patients thereafter led Cerletti and his team to believe that ECT was a panacea for psychiatric illness. ${ }^{6}$ In fact, eager physicians began recommending the procedure for nonpsychiatric conditions like psoriasis and ulcers. ${ }^{5}$ While these preliminary human tests revealed the potential for ECT in treating mental illness, the excitement quickly faded soon after.

\section{RISE AND FALL OF A SOMATIC THERAPY}

With minimal ECT regulations during the early 1940s, patient safety was a problem, which contributed to a rapid decline in ECT popularity. The first patients to undergo this treatment suffered spine fractures and joint dislocations due to the violent convulsions they experienced, which created further medical problems for physicians and a fear of ECT treatment for patients. ${ }^{3,5}$ Furthermore, adequate empirical research was minimal due to ethical limitations, with the majority of case information derived from anecdotal evidence. ${ }^{4}$ Understandably, this lack of appropriate regulation pro- 
voked improper ECT practice and therefore a poor reputation of the treatment in the eyes of the public. ${ }^{6}$ Therapy without proper indication, inadequate adjuvant therapy, and technical inconsistencies rendered the practice of ECT problematic.

After addressing initial problems of patient injury, practitioners continued to provide ECT therapy through to the 1960s until social backlash and alternative therapies caused ECT treatments to decline. ${ }^{4}$ Methodology of ECT practice incorporating anesthesia, oxygen, and muscle relaxants reduced therapy-related injuries and side effects to improve patients' desirability of this treatment. ${ }^{4}$ Still, public perception of ECT remained tainted due to the flaws observed in its early practice. These troubles became compounded by the abuse of ECT in psychiatric hospitals, which would later inspire the anti-ECT movement. Reports of ECT being used to punish patients in psychiatric institutions, as heavily publicized in the movie "One Flew Over the Cuckoo's Nest," added to the public's disapproval of ECT. ${ }^{7}$ Visuals of involuntary patients being strapped down and forced into convulsions propagated the belief that the practice was inhumane. The suicide of Ernest Hemingway, who after multiple ECT sessions to ease his depression shot himself because he found the memory loss that came with the treatment simply unbearable, added fuel to the anti-ECT movement. ${ }^{7}$ It also led to an increasing number of lawsuits against ECT professionals by former patients. ${ }^{3,8}$

During the mid-20th century, ECT remained the predominant somatic therapy available for psychiatric disorders, however the concurrent discovery and development of psychotropic medications reduced the need for convulsions. ${ }^{7}$ Medications were proving their efficacy against placebos, and pharmacotherapy was seen as more humane regardless of the side effects that coexisted. ${ }^{7}$ Consequently, pharmaceuticals emerged as the standard therapy for preliminary management of psychiatric disorders by medical professionals, driving ECT towards obsolescence. ${ }^{7}$ Unfortunately, patients with disorders resistant to pharmaceutical therapy were left lacking a proper alternative.

\section{RESURGENCE OF ECT AND INCORPORATION}

The renaissance of electroconvulsive therapy in North America was led by the American Psychiatric Association (APA) as greater empirical evidence surfaced illustrating its benefit. After nearly disappearing from practice, a surge of research and press releases from the APA helped standardize the methodology of ECT, with appropriate indications and contraindications. ${ }^{79}$ ECT re-entered the armamentarium of psychiatrists for incapacitating mental disorders such as major depression, bipolar disorder, and catatonia, where pharmacotherapy alone lacked effectiveness. ${ }^{9}$ Further research revealed nonpsychiatric potential in Parkinson's disease and epilepsy, thereby increasing its therapeutic value., ${ }^{4,9}$ Coincidentally, ECT has re-emerged as an efficacious therapeutic modality for psychiatric and nonpsychiatric disorders, as was alleged by physicians during its inception.

Since Cerletti's innovative discovery of ECT in the 1930s, the procedure has undergone a complete transformation. The technique itself has undergone changes in the equipment used to in- duce the electric stimulation, and concomitant therapies have been integrated to mitigate side effects and potential injury., ${ }^{4,7}$ Likewise, policy has adapted to prioritize the health of the patient, ensuring patient safety and other issues that had plagued the therapy earlier in its history. In contrast to the unknowing patients that ECT was tested on, patients now undergo rigorous testing by physicians to ensure that treatment is the ideal option, and are well informed of the procedure and risks. ${ }^{4}$

Still, public perception remains skewed in judging ECT as an inhumane treatment, and many individuals think the procedure is unlawful. This prevailing misconception elucidates the necessity to educate the public about the benefits of this treatment, and explains the regular press releases from the APA educating doctors. ${ }^{7}$ Currently, most patients with major depression still reject ECT as a possible treatment, despite medical literature and patient testimonials reporting positive outcomes. ${ }^{7}$ Interestingly, even $7 \%$ of physicians in 2005 believed ECT to be obsolete. ${ }^{7}$ Consequently, the APA has designated a task force of professionals to tackle ECT misinformation through press releases, workshops, and published reports. Accordingly, this effort has created an initiative to reclassify ECT as a low risk procedure..$^{10,11}$

\section{CONCLUSION}

Although electroconvulsive therapy is back in the psychiatry toolkit, its controversial history has left it entrenched in public doubt and skepticism. The misconceptions surrounding the practice are deep-rooted and will require extensive education of physicians and the public to dismantle. Organizations such as the APA have made it a priority to better educate the physicians offering ECT treatment and to eliminate the stigma associated with ECT.

Psychiatric disorders are a major burden to society, both economically and socially, and so it is critical that we continue to strive for better treatments and ultimately a cure. Consequently, research regarding the mechanisms of ECT have now yielded more effective and less invasive techniques. Newer brain stimulating techniques such as transcranial magnetic stimulation and deep brain stimulation have sprouted from the knowledge of ECT, and now provide better alternatives to convulsive therapy. ${ }^{12}$ Despite being less than a century old, ECT has survived a controversial and tortuous life, and the future may be just as uncertain. But regardless of what the future may bring for the treatment of psychiatric disorders, it is important to appreciate the influence of ECT in the present era, and the undeniable effect it has had on the treatment of mental illness in the past.

\section{REFERENCES}

1. Shorter E. History of psychiatry. Current Opinion in Psychiatry. 2008;21(6):593-597.

2. American Psychological Association. A home away from home [Internet]. 2012. Available from: http://www.apa.org/monitor/2012/03/ asylums.aspx

3. The History of Shock Therapy in Psychiatry [Internet]. Library.law. columbia.edu. 2017. Available from: http://library.law.columbia.edu/ urlmirror/CJAL/14CJALl/shock_i.htm 
4. Payne N, Prudic J. Electroconvulsive Therapy: Part I. A Perspective on the Evolution and Current Practice of ECT. Journal of Psychiatric Practice. 2009;15(5):346-368.

5. N.S. E. The origins of electroconvulsive therapy (ECT). Convulsive Therapy. 1988;4(1):5-23.

6. Ottosson J. Use and misuse of electroconvulsive treatment. Biological Psychiatry. 1985;20(9):933-946.

7. Payne N, Prudic J. Electroconvulsive Therapy: Part II. A Perspective on the Evolution and Current Practice of ECT. Journal of Psychiatric Practice. 2009;15(5):346-368.

8. Journal of the American Medical Association. Consensus conference. Electroconvulsive therapy. 1985.

9. Canadian Psychiatric Association. Electroconvulsive Therapy. 2001

10. American Psychiatric Association. APA Task Force Report on Electroconvulsive Therapy. APA; 1995.

11. American Psychiatric Association. Time is Now to Support the ECT Reclassification Effort. 2016.

12. George M, Nahas Z, Li X, et al. Novel treatments of mood disorders based on brain circuitry (ECT, MST, TMS, VNS, DBS). Seminars in Clinical Neuropsychiatry. 2002;7(4):293-304. 